\title{
EXISTENCE AND BIFURCATION RESULTS FOR FOURTH-ORDER ELLIPTIC EQUATIONS INVOLVING TWO CRITICAL SOBOLEV EXPONENTS
}

\author{
D. A. KANDILAKIS \\ Department of Sciences, Technical University of Crete, 73100 Chania, Greece \\ e-mail:dimkand@gmail.com \\ M. MAGIROPOULOS \\ Department of Sciences, Technological and Educational Institute of Crete, 71500 Heraklion, Greece \\ e-mail: mageir@stef.teiher.gr \\ and N. ZOGRAPHOPOULOS \\ Department of Sciences, Technical University of Crete, 73100 Chania, Greece \\ e-mail:nzogr@science.tuc.gr
}

(Received 28 March 2008; accepted 29 May 2008)

\begin{abstract}
Let $\Omega$ be a smooth bounded domain in $R^{N}$, with $N \geq 5$. We provide existence and bifurcation results for the elliptic fourth-order equation $\Delta^{2} u-\Delta_{p} u=$ $f(\lambda, x, u)$ in $\Omega$, under the Dirichlet boundary conditions $u=0$ and $\nabla u=0$. Here $\lambda$ is a positive real number, $1<p \leq 2^{\#}$ and $f(., ., u)$ has a subcritical or a critical growth $s, 1<s \leq 2^{*}$, where $2^{*}:=\frac{2 N}{N-4}$ and $2^{\#}:=\frac{2 N}{N-2}$. Our approach is variational, and it is based on the mountain-pass theorem, the Ekeland variational principle and the concentration-compactness principle.
\end{abstract}

AMS Subject Classification. 35J35, 35B33, 35G20, 35B32.

1. Introduction. An approach for confronting second-order critical semilinear elliptic equations in a bounded domain $\Omega$ in $R^{N}$ was introduced in [2], where it was shown that the Palais-Smale compactness condition holds for certain levels of the associated functional. Therefore, under the appropriate assumptions, the mountainpass theorem could be applied to yield a solution to the critical problem.

The existence of solutions of fourth-order critical elliptic problems can also be proved by using this approach, see $[4,5,8,11,15]$ and the references therein.

In this paper, we study problems of the form

$$
\left.\begin{array}{l}
\Delta^{2} u-\Delta_{p} u=f(\lambda, x, u) \text { in } \Omega \\
u=0, \nabla u=0 \text { on } \partial \Omega
\end{array}\right\}
$$

where $\Omega$ is a smooth bounded domain in $R^{N}$, with $N \geq 5, \Delta^{2} u$ is the biharmonic operator, $\Delta_{p} u:=\operatorname{div}\left(|\nabla u|^{p-2} \nabla u\right)$ is the $p$-Laplace operator, $f: R \times \Omega \times R \rightarrow R$ is a function with either subcritical or critical growth in the third variable and $\lambda$ is a positive real number.

Problem (1) has not been addressed in such a general context before. A similar problem was examined by [6], [12] and [16], who studied not the difference, but the 
sum of the biharmonic and the $p$-Laplace operator for the case $p=2$ and with Navier boundary conditions.

Owing to the presence of the biharmonic and $p$-Laplace operators in the equation, two critical exponents could appear: the critical exponent $2^{*}:=\frac{2 N}{N-4}$ for the Sobolev embedding $H_{0}^{2}(\Omega) \hookrightarrow L^{q}(\Omega)$ and the critical exponent $2^{\#}:=\frac{2 N}{N-2}$ for the Sobolev embedding $H_{0}^{1}(\Omega) \hookrightarrow L^{q}(\Omega)$. Our purpose is to provide solutions for the subcritical and critical cases, which arise as $s$, the growth of $f$ in the third variable, varies between 1 and $2^{*}$ and $p$ varies between 1 and $2^{\#}$. These solutions will be found as the critical points of the Frechet differentiable energy functional given by

$$
\Phi_{\lambda}(u):=\frac{1}{2} \int_{\Omega}(\Delta u)^{2} d x+\frac{1}{p} \int_{\Omega}|\nabla u|^{p} d x-\int_{\Omega} \int_{0}^{u} f(\lambda, x, s) d s d x,
$$

which is defined on the Sobolev space $E:=H_{0}^{2}(\Omega)$ endowed with the equivalent norm

$$
\|u\|_{E}^{2}=\int_{\Omega}(\Delta u)^{2}
$$

We now present our results. In Section 2, we examine the subcritical case where $f(\lambda, x, u)=\lambda|u|^{s-2} u, 1<p<2^{\#}$ and $1<s<2^{*}$, and prove the following:

THEOREM 1. Let $1<p<2^{\#}$.

(i) Suppose that $2<s<2^{*}$. Then if $p<s$, (1) admits a solution for every $\lambda>0$, while if $s \leq p$, there exists $\lambda_{0}>0$ such that (1) admits a solution for every $\lambda>\lambda_{0}$.

(ii) Suppose that $1<s<2$. Then if $s<p$, (1) admits a solution for every $\lambda>0$, while if $p<s$, there exists $\lambda_{0}>0$ such that (1) admits a solution for every $\lambda>\lambda_{0}$.

(iii) If $\lambda>\lambda_{1}, s=2$ and $2<p<2^{\#}$, then (1) admits a solution.

Here $\lambda_{1}$ denotes the first eigenvalue of $\Delta^{2}$ with Dirichlet boundary conditions.

In Section 3, we examine the subcritical case for $s$ and the critical case $p=2^{\#}$. We show the following:

THEOREM 2. (i) If $p=2^{\#}$ and $2<s<2^{\#}$, then there exists $\widehat{\lambda}>0$ such that (1) admits a nontrivial solution for every $\lambda>\widehat{\lambda}$.

(ii) If $p=2^{\#}$ and $2^{\#}<s<2^{*}$, then (1) admits a solution for every $\lambda>0$.

In Section 4, in an effort to extend our results to the critical case $s=2^{*}$, we assume that $f(\lambda, x, u)=\lambda|u|^{2^{*}-2} u+g(x)$, where $g: \Omega \rightarrow R$ is a nontrivial continuous function, and in this situation, we obtain:

THEOREM 3. If $\|g\|_{\frac{2 N}{N+4}}$ is small enough, then (18) admits a solution.

Here, $p$ is restricted in the interval $\left(1,2^{\#}\right)$, and it is an open question whether there is a solution if $p=2^{\#}$.

Finally, in Section 5, we study the bifurcation properties for the problem

$$
\left.\begin{array}{l}
\Delta^{2} u-\Delta_{p} u=\lambda u+h(x, \lambda)|u|^{2^{*}-2} u \text { in } \Omega, \\
u=0, \nabla u=0 \text { on } \partial \Omega,
\end{array}\right\}
$$

where $1<p<2^{\#}$, and we have the following: 
THEOREM 4. Equation (2) admits a continuum $C$ of nontrivial solutions $(\lambda, u) \subseteq$ $R \times E$ bifurcating from $\left(\lambda_{1}, 0\right)$, which meets the boundary of $\left[\lambda_{1}-d, \lambda_{1}+d\right] \times B\left(0, \rho_{0}\right)$.

2. The subcritical case. In this section, we assume that $f(\lambda, x, u)=\lambda|u|^{s-2} u, 1<$ $p<2^{\#}$ and $1<s<2^{*}$.

LEMMA 5. Suppose that one of the following statements holds:

(i) $1<p<2^{\#}, s \in\left(1,2^{*}\right) \backslash\{2\}$ and $\lambda>0$.

(ii) $s=2,2<p<2^{\#}$ and $\lambda>0$.

(iii) $s=2,1<p \leq 2$ and $\lambda<\lambda_{1}$.

Then $\Phi_{\lambda}($.$) satisfies the Palais-Smale condition.$

Proof. Assume first that $2 \leq p<2^{\#}$. Let $\left\{u_{n}\right\}_{n \in N}$ be a Palais-Smale sequence, that is,

(i) $\Phi_{\lambda}\left(u_{n}\right)$ is bounded and

(ii) $\Phi_{\lambda}^{\prime}\left(u_{n}\right) \rightarrow 0$.

From (i), there exists $M>0$ such that

$$
-M \leq \frac{1}{2} \int_{\Omega}\left(\Delta u_{n}\right)^{2}+\frac{1}{p} \int_{\Omega}\left|\nabla u_{n}\right|^{p}-\frac{\lambda}{s} \int_{\Omega}\left|u_{n}\right|^{s} \leq M,
$$

while (ii) implies that

$$
\int_{\Omega}\left(\Delta u_{n}\right)^{2}+\int_{\Omega}\left|\nabla u_{n}\right|^{p}-\lambda \int_{\Omega}\left|u_{n}\right|^{s}=o_{n}(1)\left\|u_{n}\right\|_{E} .
$$

Multiplying (4) by $-1 / a, a>0$, and adding memberwise to (3), we obtain

$$
\begin{gathered}
-M-o_{n}(1)\left\|u_{n}\right\|_{E} \\
\leq\left(\frac{1}{2}-\frac{1}{a}\right) \int_{\Omega}\left(\Delta u_{n}\right)^{2}+\left(\frac{1}{p}-\frac{1}{a}\right) \int_{\Omega}\left|\nabla u_{n}\right|^{p}+\lambda\left(\frac{1}{a}-\frac{1}{s}\right) \int_{\Omega}\left|u_{n}\right|^{s} \\
\leq M-o_{n}(1)\left\|u_{n}\right\|_{E} .
\end{gathered}
$$

By taking $a=p$ in (5), the boundedness of $\left\|u_{n}\right\|_{E}$ is straightforward for the case $p \leq s$. For $s<p$, we take $a>p$ and exploit the embeddings $H_{0}^{1}(\Omega) \hookrightarrow L^{s}(\Omega)$ and $\left(L^{p}(\Omega)\right)^{N} \hookrightarrow\left(L^{2}(\Omega)\right)^{N}$ to get

$$
\begin{gathered}
\left(\frac{1}{2}-\frac{1}{a}\right) \int_{\Omega}\left(\Delta u_{n}\right)^{2}+\left(\frac{1}{p}-\frac{1}{a}\right) \int_{\Omega}\left|\nabla u_{n}\right|^{p}+\lambda c\left(\frac{1}{a}-\frac{1}{s}\right)\left(\int_{\Omega}\left|\nabla u_{n}\right|^{p}\right)^{\frac{s}{p}} \\
\leq M-o_{n}(1)\left\|u_{n}\right\|_{E}
\end{gathered}
$$

from where we obtain once more the desired boundedness. Obvious modifications of the same idea yields boundedness for the rest of the cases. 
Thus, we may assume that, up to a subsequence, $u_{n} \rightarrow u$ weakly in $E$. From the Sobolev embedding, we obtain that

$$
\left.\begin{array}{l}
\Delta u_{n} \rightarrow \Delta u \text { weakly in } L^{2}(\Omega) \\
u_{n} \rightarrow u \text { in } L^{s}(\Omega) \text { and } \\
\nabla u_{n} \rightarrow \nabla u \text { in }\left(L^{p}(\Omega)\right)^{N}
\end{array}\right\}
$$

By $(4), \Phi_{\lambda}^{\prime}\left(u_{n}\right)\left(u_{n}\right) \rightarrow 0$, that is,

$$
\int_{\Omega}\left(\Delta u_{n}\right)^{2}+\int_{\Omega}\left|\nabla u_{n}\right|^{p}-\lambda \int_{\Omega}\left|u_{n}\right|^{s} \rightarrow 0
$$

and so

$$
\int_{\Omega}\left(\Delta u_{n}\right)^{2} \rightarrow \lambda \int_{\Omega}|u|^{s}-\int_{\Omega}|\nabla u|^{p}
$$

On the other hand, since $\Phi_{\lambda}^{\prime}\left(u_{n}\right)(u) \rightarrow 0$,

$$
\int_{\Omega}\left(\Delta u_{n}\right)(\Delta u)+\int_{\Omega}\left|\nabla u_{n}\right|^{p-2} \nabla u_{n} \nabla u-\lambda \int_{\Omega}\left|u_{n}\right|^{s-2} u_{n} u \rightarrow 0 .
$$

Combining (6)-(8), we conclude that

$$
\int_{\Omega}(\Delta u)^{2}=\lambda \int_{\Omega}|u|^{s}-\int_{\Omega}|\nabla u|^{p}
$$

Consequently, $\left\|u_{n}\right\|_{E} \rightarrow\|u\|_{E}$. The uniform convexity of $E$ implies that $u_{n} \rightarrow u$ in $E$.

Proof of Theorem 1. (i) Assume first that $2 \leq p<s$. By the Sobolev embedding, if $\|u\|_{E}$ is sufficiently small, then

$$
\Phi_{\lambda}(u) \geq \frac{1}{2} \int_{\Omega}(\Delta u)^{2}+\frac{1}{p} \int_{\Omega}|\nabla u|^{p}-d\left(\int_{\Omega}(\Delta u)^{2}\right)^{\frac{s}{2}}>\delta
$$

for some $d, \delta>0$. Note that for $u \neq 0$,

$$
\Phi_{\lambda}(t u)=\frac{t^{2}}{2} \int_{\Omega}(\Delta u)^{2}+\frac{t^{p}}{p} \int_{\Omega}|\nabla u|^{p}-\frac{\lambda t^{s}}{s} \int_{\Omega}|u|^{s} \rightarrow-\infty
$$

as $t \rightarrow \infty$. Applying the mountain-pass theorem we get a solution to (1).

Suppose next that $2<s \leq p$. We define

$$
\lambda_{0}:=\inf _{u \in E \backslash\{0\}} \frac{\frac{1}{2} \int_{\Omega}(\Delta u)^{2}+\frac{1}{p} \int_{\Omega}|\nabla u|^{p}}{\frac{1}{s} \int_{\Omega}|u|^{s}} .
$$


The continuity of the embedding $H_{0}^{1}(\Omega) \hookrightarrow L^{t}(\Omega), t \in\left(1,2^{\#}\right]$ implies that for every $u \in E \backslash\{0\}$,

$$
\begin{gathered}
\frac{\frac{1}{2} \int_{\Omega}(\Delta u)^{2}+\frac{1}{p} \int_{\Omega}|\nabla u|^{p}}{\frac{1}{s} \int_{\Omega}|u|^{s}} \geq \frac{c_{1}\left(\int_{\Omega}|\nabla u|^{p}\right)^{\frac{2}{p}}+\frac{1}{p} \int_{\Omega}|\nabla u|^{p}}{c_{2}\left(\int_{\Omega}|\nabla u|^{p}\right)^{\frac{s}{p}}} \\
=\frac{c_{1}}{c_{2}}\left(\int_{\Omega}|\nabla u|^{p}\right)^{\frac{2-s}{p}}+\frac{1}{p c_{2}}\left(\int_{\Omega}|\nabla u|^{p}\right)^{\frac{p-s}{p}}>\eta
\end{gathered}
$$

for some $\eta, c_{1}, c_{2}>0$. Thus, $\lambda_{0}>0$. Consequently, if $\lambda>\lambda_{0}$, there exists $u_{\lambda} \in E \backslash\{0\}$ such that

$$
\frac{1}{2} \int_{\Omega}\left(\Delta u_{\lambda}\right)^{2}+\frac{1}{p} \int_{\Omega}\left|\nabla u_{\lambda}\right|^{p}<\frac{\lambda}{s} \int_{\Omega}\left|u_{\lambda}\right|^{s}
$$

and so $\Phi_{\lambda}\left(u_{\lambda}\right)<0$. Since (9) guarantees that $\Phi_{\lambda}($.) is positive close to the origin, the mountain-pass theorem provides a solution to (1).

Now let $1<p<2$. In view of the embedding $E \hookrightarrow L^{s}(\Omega)$, we have

$$
\Phi_{\lambda}(u) \geq \frac{1}{2} \int_{\Omega}(\Delta u)^{2}-d\left(\int_{\Omega}(\Delta u)^{2}\right)^{\frac{s}{2}}
$$

for some $d>0$, which implies that $\Phi_{\lambda}($.$) is positive near the origin. Since$ $\lim _{t \rightarrow+\infty} \Phi_{\lambda}(t u)=-\infty$, the mountain-pass theorem provides a solution to (1).

(ii) Assume first that $s<p$. In view of the embedding $E \hookrightarrow L^{s}(\Omega)$, we have

$$
\Phi_{\lambda}(u) \geq d\left(\int_{\Omega}|u|^{s}\right)^{\frac{2}{s}}-\frac{\lambda}{s} \int_{\Omega}|u|^{s}
$$

for some $d>0$ and so $\Phi_{\lambda}($.$) is bounded below. Since \Phi_{\lambda}($.$) satisfies the Palais-Smale$ condition, Ekeland's variational principle [9] provides a solution to (1), which is nontrivial because $\Phi_{\lambda}($.) assumes negative values near the origin.

Let now $1<p \leq s<2$. Then $\Phi_{\lambda}($.) satisfies the Palais-Smale condition and is bounded below. If $\lambda>\lambda_{0}$, in view of (11) and (12), $\Phi_{\lambda}($.$) assumes negative values and$ so Ekeland's variational principle provides a nontrivial solution to (1).

(iii) By exploiting the embedding $W_{0}^{1, p}(\Omega) \hookrightarrow L^{2}(\Omega)$, we get

$$
\begin{aligned}
\Phi_{\lambda}(u) & =\frac{1}{2} \int_{\Omega}(\Delta u)^{2}+\frac{1}{p} \int_{\Omega}|\nabla u|^{p}-\frac{\lambda}{2} \int_{\Omega}|u|^{2} \\
& \geq \frac{1}{2}\left(\lambda_{1}-\lambda\right) \int_{\Omega}|u|^{2}+\frac{1}{p} \int_{\Omega}|\nabla u|^{p} \\
& \geq \frac{1}{2}\left(\lambda_{1}-\lambda\right) \int_{\Omega}|u|^{2}+d\left(\int_{\Omega}|u|^{2}\right)^{\frac{p}{2}}
\end{aligned}
$$

for some $d>0$. Thus, $\Phi_{\lambda}($.$) is bounded below. Also, for an eigenfunction u_{1}$ corresponding to $\lambda_{1}$ and $t>0$ sufficiently small,

$$
\Phi_{\lambda}\left(t u_{1}\right)=\frac{t^{2}}{2}\left(1-\frac{\lambda}{\lambda_{1}}\right) \int_{\Omega}\left(\Delta u_{1}\right)^{2}+\frac{t^{p}}{p} \int_{\Omega}\left|\nabla u_{1}\right|^{p}<0 .
$$


Since $\Phi_{\lambda}($.$) also satisfies the Palais-Smale condition, Ekeland's variational principle$ provides a solution to (1).

\section{The critical case $p=2^{\#}$.}

Proof of Theorem 2. (i) Let $p_{n} \in\left(s, 2^{\#}\right)$, with $p_{n} \rightarrow 2^{\#}$. Theorem 1 guarantees that there exists $\lambda_{n}>0$ such that (1) admits a solution for every $\lambda>\lambda_{n}$. The Sobolev embedding implies that the sequence $\left\{\lambda_{n}\right\}_{n \in N}$ is bounded. Define $\widehat{\lambda}:=\sup _{n \rightarrow+\infty} \lambda_{n}$. Thus, for $\lambda>\widehat{\lambda}$, there exists $u_{n} \in E$ such that

$$
\frac{1}{2} \int_{\Omega}\left(\Delta u_{n}\right)^{2}+\frac{1}{p_{n}} \int_{\Omega}\left|\nabla u_{n}\right|^{p_{n}}=\frac{\lambda}{s} \int_{\Omega}\left|u_{n}\right|^{s} .
$$

The embeddings $H_{0}^{1}(\Omega) \hookrightarrow L^{s}(\Omega)$ and $L^{p_{n}}(\Omega) \hookrightarrow L^{2}(\Omega)$ imply that

$$
\|u\|_{L^{s}(\Omega)} \leq c\|\nabla u\|_{L^{2}(\Omega)} \text { and }\|\nabla u\|_{L^{2}(\Omega)} \leq c_{n}\|\nabla u\|_{L^{p^{n}}(\Omega)},
$$

where $\left\{c_{n}\right\}_{n \in N}$ is a bounded sequence. Thus,

$$
\|u\|_{L^{s}(\Omega)} \leq d\|\nabla u\|_{L^{p^{n}(\Omega)}}
$$

for some $d>0$. Combining (14) and (15), we see that $\left\|\nabla u_{n}\right\|_{L^{p_{n}(\Omega)}}, n \in N$, is bounded. By (14), we conclude that the sequence $\left\{\left\|u_{n}\right\|_{E}\right\}_{n \in N}$ is bounded. By passing to a subsequence, if necessary, we may assume that $u_{n} \rightarrow u$ weakly in $E$. Thus, for $\psi \in C_{0}^{\infty}(\Omega)$ and $\lambda>\widehat{\lambda}$, we have

$$
\int_{\Omega} \Delta u_{n} \Delta \psi+\int_{\Omega}\left|\nabla u_{n}\right|^{p_{n}-2} \nabla u_{n} \nabla \psi=\lambda \int_{\Omega}\left|u_{n}\right|^{s-2} u_{n} \psi
$$

for every $n \in N$. It is clear that

$$
\begin{gathered}
\int_{\Omega} \Delta u_{n} \Delta \psi \rightarrow \int_{\Omega} \Delta u \Delta \psi, \\
\int_{\Omega}\left|u_{n}\right|^{s-2} u_{n} \psi \rightarrow \int_{\Omega}|u|^{s-2} u \psi,
\end{gathered}
$$

while Theorem IV.9 in [1] yields

$$
\int_{\Omega}\left|\nabla u_{n}\right|^{p_{n}-2} \nabla u_{n} \nabla \psi \rightarrow \int_{\Omega}|\nabla u|^{2^{\#}-2} \nabla u \nabla \psi
$$

Thus,

$$
\int_{\Omega} \Delta u \Delta \psi+\int_{\Omega}|\nabla u|^{2^{\#}-2} \nabla u \nabla \psi=\lambda \int_{\Omega}|u|^{s-2} u \psi,
$$

that is, $u$ is a solution to (1), with $p=2^{\#}$. We show that $u \neq 0$. Indeed, if we assume that $u_{n} \rightarrow 0$ in $E$, then for the sequence $v_{n}:=\frac{u_{n}}{\left\|u_{n}\right\|_{E}}, n \in N$, we would have

$$
1=\int_{\Omega}\left(\Delta v_{n}\right)^{2}=\lambda\left\|u_{n}\right\|_{E}^{s-2} \int_{\Omega}\left|v_{n}\right|^{s}-\left\|u_{n}\right\|_{E}^{p_{n}-2} \int_{\Omega}\left|\nabla v_{n}\right|^{p_{n}} \rightarrow 0,
$$


a contradiction.

(ii) Assume that $E$ is supplied with the norm

$$
\|u\|=\left(\int_{\Omega}(\Delta u)^{2}\right)^{\frac{1}{2}}+\left(\int_{\Omega}|\nabla u|^{2^{\#}}\right)^{\frac{1}{2^{\#}}} .
$$

We show that $\Phi_{\lambda}\left(\right.$.) satisfies the Palais-Smale condition. Let $\left\{u_{n}\right\}_{n \in N}$ be a Palais-Smale sequence. Working as in Lemma 5, we see that $\left\{u_{n}\right\}_{n \in N}$ is bounded in $E$ with respect to the norm $\|\mid\| \|$. Therefore, by passing to a subsequence, if necessary, we may assume that $u_{n} \rightarrow u$ weakly in $E$ and $W_{0}^{1,2^{\#}}(\Omega)$. Since $\Phi_{\lambda}^{\prime}\left(u_{n}\right)\left(u_{n}\right) \rightarrow 0$ and $\Phi_{\lambda}^{\prime}\left(u_{n}\right)(u) \rightarrow 0$, we have

$$
\int_{\Omega}\left(\Delta u_{n}\right)^{2}+\int_{\Omega}\left|\nabla u_{n}\right|^{2^{\#}} \rightarrow \lambda \int_{\Omega}|u|^{s}
$$

and

$$
\int_{\Omega}\left(\Delta u_{n}\right)(\Delta u)+\int_{\Omega}\left|\nabla u_{n}\right|^{2^{\#}-2} \nabla u_{n} \nabla u \rightarrow \lambda \int_{\Omega}|u|^{s} .
$$

Note that $\nabla u_{n} \rightarrow \nabla u$ in $L^{2^{\#}-2}(\Omega)$ and $\Delta u_{n} \rightarrow \Delta u$ weakly, so (17) yields

$$
\int_{\Omega}(\Delta u)^{2}+\int_{\Omega}|\nabla u|^{2^{\#}}=\lambda \int_{\Omega}|u|^{s},
$$

and this fact combined with (16) shows that $u_{n} \rightarrow u$ in $E$ and $W^{1,2^{\#}}(\Omega)$. By (13), $\Phi_{\lambda}($.) is positive near the origin. Since $\lim _{t \rightarrow+\infty} \Phi_{\lambda}(t u)=-\infty$, the mountain-pass theorem provides a solution to (1).

4. The critical case $s=2^{*}$. In this section, we study the nonhomogeneous equation

$$
\Delta^{2} u-\Delta_{p} u=\lambda|u|^{2^{*}-2} u+g \text { in } \Omega
$$

subject to the Dirichlet boundary conditions, where $g: \Omega \rightarrow R$ is a nontrivial continuous function and $\lambda>0$. We follow the approach of Guedda [11].

The energy functional associated to (18) is

$$
\Psi_{\lambda}(u):=\frac{1}{2} \int_{\Omega}(\Delta u)^{2} d x+\frac{1}{p} \int_{\Omega}|\nabla u|^{p} d x-\frac{\lambda}{2^{*}} \int_{\Omega}|u|^{2^{*}} d x-\int_{\Omega} g u .
$$

Let $S:=\inf \left\{\|u\|_{E}^{2}:\|u\|^{2^{*}}=1\right\}$ be the best constant in the Sobolev inclusion $H_{0}^{2}(\Omega) \subset$ $L^{2^{*}}(\Omega)$. By Theorem 2.1 in $[\mathbf{8}], S$ is attained by the functions $u_{\varepsilon}$ given by

$$
u_{\varepsilon}(x):=K_{N}\left(\frac{\varepsilon}{\varepsilon^{2}+\left|x-x_{0}\right|^{2}}\right)^{\frac{N-4}{2}}
$$

where

$$
K_{N}:=[(N-4)(N-2) N(N+2)]^{\frac{N-4}{8}}
$$


for any $\varepsilon>0$ and $x_{0} \in R^{N}$. Furthermore, the functions $u_{\varepsilon}$, with $x_{0}=0$, are the only positive spherically symmetric solutions of the equation

$$
\Delta^{2} u=u^{\frac{N+4}{N-4}} \text { in } R^{N}
$$

which are decreasing in $|x|$.

Lemma 6. Suppose that $1<p<2^{\#}$. Then $\Psi_{\lambda}($.$) satisfies a local Palais-Smale$ condition in the strip $\left(-\infty, \frac{2 \lambda}{N}\left(\frac{S}{\lambda}\right)^{\frac{N}{4}}-K\right)$, where

$$
K:=\frac{\left(2^{*}-1\right)\left(2^{\#}-1\right)^{\eta}\|g\|_{\eta}^{\eta}}{\lambda^{\eta-1}\left(2^{*}-2^{\#}\right)^{\eta-1} 2^{*} 2^{\#}} \text { and } \eta:=\frac{2 N}{N+4} .
$$

Proof. Assume that $\lim _{n \rightarrow+\infty} \Psi_{\lambda}\left(u_{n}\right)=\alpha<\frac{2 \lambda}{N}\left(\frac{S}{\lambda}\right)^{\frac{N}{4}}-K$ and $\Psi_{\lambda}^{\prime}\left(u_{n}\right) \rightarrow 0$ in $E^{*}$. Then,

$$
\frac{1}{2} \int_{\Omega}\left(\Delta u_{n}\right)^{2}+\frac{1}{p} \int_{\Omega}\left|\nabla u_{n}\right|^{p}-\frac{\lambda}{2^{*}} \int_{\Omega}\left|u_{n}\right|^{2^{*}}-\int_{\Omega} g u_{n}=\alpha+o_{n}(1)
$$

and

$$
\int_{\Omega}\left(\Delta u_{n}\right)^{2}+\int_{\Omega}\left|\nabla u_{n}\right|^{p}-\lambda \int_{\Omega}\left|u_{n}\right|^{2^{*}}-\int_{\Omega} g u_{n}=o_{n}(1)\left\|u_{n}\right\|_{E} .
$$

Combining (22) and (23), we get

$$
\begin{aligned}
& \left(\frac{1}{2}-\frac{1}{2^{*}}\right) \int_{\Omega}\left(\Delta u_{n}\right)^{2}+\left(\frac{1}{p}-\frac{1}{2^{*}}\right) \int_{\Omega}\left|\nabla u_{n}\right|^{p}-\left(1-\frac{1}{p}\right) \int_{\Omega} g u_{n} \\
& \quad=\alpha+o_{n}(1)+o_{n}(1)\left\|u_{n}\right\|_{E},
\end{aligned}
$$

which implies that $\left\{u_{n}\right\}_{n \in N}$ is bounded in $E$. By passing to a subsequence, if necessary, we have that $u_{n} \rightarrow u$ weakly in $E$. In view of the Sobolev embedding and the concentrationcompactness principle [13],

$$
\begin{gathered}
u_{n} \rightarrow u \text { in } L^{2}(\Omega) \text { and a.e. in } \bar{\Omega} \\
\nabla u_{n} \rightarrow \nabla u \text { in } L^{q}(\Omega)^{N}, 1<q<2^{\#}, \text { and a.e. in } \bar{\Omega} \\
\left|u_{n}\right|^{2^{*}} \rightarrow v=|u|^{2^{*}}+\sum_{j \in J} v_{j} \delta_{x_{j}} \text { in the } w^{*} \text { - sense, } \\
\left(\Delta u_{n}\right)^{2} \rightarrow \mu \geq(\Delta u)^{2}+\sum_{j \in J} \mu_{j} \delta_{x_{j}} \text { in the } w^{*} \text { - sense, } \\
S v_{j}^{\frac{2}{2^{*}}} \leq \mu_{j},
\end{gathered}
$$

where $J$ is a finite set and $x_{j} \in \bar{\Omega}$. We show that $\nu_{j}=\mu_{j}=0$ for every $j \in J$. For a fixed $j \in J$ and $\varepsilon>0$, let $\varphi \in C_{0}^{\infty}\left(R^{N}\right)$ such that

$$
\left.\begin{array}{c}
0 \leq \varphi \leq 1, \varphi=1 \text { on } B\left(x_{j}, \varepsilon\right), \varphi=0 \text { on } R^{N} \backslash B\left(x_{j}, 2 \varepsilon\right), \\
|\nabla \varphi| \leq \frac{2}{\varepsilon} \text { and }|\Delta \varphi| \leq \frac{2}{\varepsilon^{2}}
\end{array}\right\}
$$

By hypothesis,

$$
\Psi_{\lambda}^{\prime}\left(u_{n}\right)\left(u_{n} \varphi \chi_{\bar{\Omega}}\right) \rightarrow 0 \text { as } n \rightarrow \infty
$$

that is,

$$
\begin{gathered}
\int_{B\left(x_{j}, 2 \varepsilon\right) \cap \Omega}\left(\Delta u_{n}\right) \Delta\left(u_{n} \varphi\right)+\int_{B\left(x_{j}, 2 \varepsilon\right) \cap \Omega}\left|\nabla u_{n}\right|^{p-2} \nabla u_{n} \nabla\left(u_{n} \varphi\right) \\
-\int_{B\left(x_{j}, 2 \varepsilon\right) \cap \Omega} g u_{n} \varphi-\lambda \int_{B\left(x_{j}, 2 \varepsilon\right) \cap \Omega}\left|u_{n}\right|^{2^{*}} \varphi \rightarrow 0
\end{gathered}
$$


In view of (24) and (25),

$$
\begin{gathered}
\int_{B\left(x_{j}, 2 \varepsilon\right) \cap \Omega}\left(\Delta u_{n}\right) \Delta\left(u_{n} \varphi\right)+\int_{B\left(x_{j}, 2 \varepsilon\right) \cap \Omega}\left|\nabla u_{n}\right|^{p-2} \nabla u_{n} \nabla\left(u_{n} \varphi\right) \\
-\int_{B\left(x_{j}, 2 \varepsilon\right) \cap \Omega} g u_{n} \varphi \rightarrow \lambda \int_{B\left(x_{j}, 2 \varepsilon\right) \cap \Omega} \varphi d \nu
\end{gathered}
$$

as $n \rightarrow+\infty$. Since

$$
\begin{aligned}
& \int_{B\left(x_{j}, 2 \varepsilon\right) \cap \Omega}\left|\nabla u_{n}\right|^{p-2} \nabla u_{n} \nabla\left(u_{n} \varphi\right) \\
& =\int_{B\left(x_{j}, 2 \varepsilon\right) \cap \Omega}\left|\nabla u_{n}\right|^{p} \varphi+\int_{B\left(x_{j}, 2 \varepsilon\right) \cap \Omega}\left|\nabla u_{n}\right|^{p-2} \nabla u_{n} \nabla \varphi u_{n} \\
& \rightarrow \int_{B\left(x_{j}, 2 \varepsilon\right) \cap \Omega}|\nabla u|^{p} \varphi+\int_{B\left(x_{j}, 2 \varepsilon\right) \cap \Omega}|\nabla u|^{p-2} \nabla u \nabla \varphi u,
\end{aligned}
$$

(26) becomes

$$
\begin{aligned}
\lim _{n \rightarrow \infty} & \int_{B\left(x_{j}, 2 \varepsilon\right) \cap \Omega}\left(\Delta u_{n}\right) \Delta\left(u_{n} \varphi\right) \\
= & \int_{B\left(x_{j}, 2 \varepsilon\right) \cap \Omega} \varphi d v-\int_{B\left(x_{j}, 2 \varepsilon\right) \cap \Omega}|\nabla u|^{p} \varphi-\int_{B\left(x_{j}, 2 \varepsilon\right) \cap \Omega}|\nabla u|^{p-2} \nabla u \nabla \varphi u \\
& -\int_{B\left(x_{j}, 2 \varepsilon\right) \cap \Omega} g u_{n} \varphi \rightarrow \lambda v_{j},
\end{aligned}
$$

as $\varepsilon \rightarrow 0$. Also,

$$
\begin{aligned}
& \int_{B\left(x_{j}, 2 \varepsilon\right) \cap \Omega}\left(\Delta u_{n}\right)\left(\Delta u_{n} \varphi\right)=\int_{B\left(x_{j}, 2 \varepsilon\right) \cap \Omega}\left(\Delta u_{n}\right)^{2} \varphi \\
& +\int_{B\left(x_{j}, 2 \varepsilon\right) \cap \Omega}\left(\Delta u_{n}\right)(\Delta \varphi) u_{n}+2 \int_{B\left(x_{j}, 2 \varepsilon\right) \cap \Omega}\left(\Delta u_{n}\right)\left(\nabla u_{n} \nabla \varphi\right) .
\end{aligned}
$$

But

$$
\lim _{n \rightarrow+\infty} \int_{B\left(x_{j}, 2 \varepsilon\right) \cap \Omega}\left(\Delta u_{n}\right)^{2} \varphi \rightarrow \int_{B\left(x_{j}, 2 \varepsilon\right) \cap \Omega} \varphi d \mu \geq \mu_{j}
$$

as $\varepsilon \rightarrow 0$,

$$
\begin{aligned}
& \lim _{n \rightarrow+\infty}\left|\int_{B\left(x_{j}, 2 \varepsilon\right) \cap \Omega}\left(\Delta u_{n}\right)(\Delta \varphi) u_{n}\right| \\
& \leq \lim _{n \rightarrow+\infty}\left[\left(\int_{B\left(x_{j}, 2 \varepsilon\right) \cap \Omega}\left|\Delta u_{n}\right|^{2}\right)^{\frac{1}{2}}\left(\int_{B\left(x_{j}, 2 \varepsilon\right) \cap \Omega}|\Delta \varphi|^{2}\left|u_{n}\right|^{2}\right)^{\frac{1}{2}}\right]
\end{aligned}
$$




$$
\begin{aligned}
& \leq c_{1}\left(\int_{B\left(x_{j}, 2 \varepsilon\right) \cap \Omega}|\Delta \varphi|^{2}|u|^{2}\right)^{\frac{1}{2}} \\
& \leq c_{1}\left(\int_{B\left(x_{j}, 2 \varepsilon\right) \cap \Omega}|\Delta \varphi|^{\frac{N}{2}}\right)^{\frac{2}{N}}\left(\int_{B\left(x_{j}, 2 \varepsilon\right) \cap \Omega}|\Delta \varphi|^{2}|u|^{2}\right)^{\frac{1}{2}}\left(\int_{B\left(x_{j}, 2 \varepsilon\right) \cap \Omega}|u|^{2^{*}}\right)^{\frac{1}{2^{*}}} \\
& \leq c_{2}\left(\int_{B\left(x_{j}, 2 \varepsilon\right) \cap \Omega}|u|^{2^{*}}\right)^{\frac{1}{2^{*}}} \rightarrow 0
\end{aligned}
$$

as $\varepsilon \rightarrow 0$, and

$$
\begin{aligned}
& \lim _{n \rightarrow+\infty}\left|\int_{B\left(x_{j}, 2 \varepsilon\right) \cap \Omega}\left(\Delta u_{n}\right)\left(\nabla u_{n} \nabla \varphi\right)\right| \\
& \leq \lim _{n \rightarrow+\infty}\left[\left(\int_{B\left(x_{j}, 2 \varepsilon\right) \cap \Omega}\left|\Delta u_{n}\right|^{2}\right)^{\frac{1}{2}}\left(\int_{B\left(x_{j}, 2 \varepsilon\right) \cap \Omega}|\nabla \varphi|^{2}\left|\nabla u_{n}\right|^{2}\right)^{\frac{1}{2}}\right] \\
& \leq c_{3}\left(\int_{B\left(x_{j}, 2 \varepsilon\right) \cap \Omega}|\nabla \varphi|^{2}|\nabla u|^{2}\right)^{\frac{1}{2}} \\
& \leq c_{3}\left(\int_{B\left(x_{j}, 2 \varepsilon\right) \cap \Omega}|\nabla \varphi|^{N}\right)^{\frac{1}{N}}\left(\int_{B\left(x_{j}, 2 \varepsilon\right) \cap \Omega}|\nabla u|^{\frac{2 N}{N-2}}\right)^{\frac{N-2}{2 N}} \\
& \quad \leq c_{4}\left(\int_{B\left(x_{j}, 2 \varepsilon\right) \cap \Omega}|\nabla u|^{\frac{2 N}{N-2}}\right)^{\frac{N-2}{2 N}} \rightarrow 0,
\end{aligned}
$$

as $\varepsilon \rightarrow 0$. Combining (27)-(31), we obtain $\mu_{j} \leq \lambda v_{j}$. By (24), $S v_{j}^{2 / 2^{*}} \leq \lambda v_{j}$, which implies that either $v_{j}=0$ or $v_{j} \geq\left(\frac{S}{\lambda}\right)^{N / 4}$. If we assume that $v_{j} \geq\left(\frac{S}{\lambda}\right)^{N / 4}$, then

$$
\begin{aligned}
\alpha= & \lim _{n \rightarrow+\infty}\left[\Psi_{\lambda}\left(u_{n}\right)-\frac{1}{2^{\#}} \Psi_{\lambda}^{\prime}\left(u_{n}\right) u_{n}\right] \\
= & \lim _{n \rightarrow+\infty}\left[\left(\frac{1}{2}-\frac{1}{2^{\#}}\right) \int_{\Omega}\left(\Delta u_{n}\right)^{2}+\left(\frac{1}{p}-\frac{1}{2^{\#}}\right) \int_{\Omega}\left|\nabla u_{n}\right|^{p}+\lambda\left(\frac{1}{2^{\#}}-\frac{1}{2^{*}}\right)\right. \\
& \left.\times \int_{\Omega}\left|u_{n}\right|^{2^{*}}\right]-\left(1-\frac{1}{2^{\#}}\right) \int_{\Omega} g u \\
\geq & \left(\frac{1}{2}-\frac{1}{2^{\#}}\right) \int_{\Omega}(\Delta u)^{2}+\left(\frac{1}{2}-\frac{1}{2^{\#}}\right) \mu_{j}+\lambda\left(\frac{1}{2^{\#}}-\frac{1}{2^{*}}\right) \int_{\Omega}|u|^{2^{*}}
\end{aligned}
$$




$$
\begin{aligned}
& +\lambda\left(\frac{1}{2^{\#}}-\frac{1}{2^{*}}\right) v_{j}-\left(1-\frac{1}{2^{\#}}\right)\|g\|_{\eta}\left(\int_{\Omega}|u|^{2^{*}}\right)^{\frac{1}{2^{*}}} \\
\geq & \frac{2 \lambda}{N}\left(\frac{S}{\lambda}\right)^{\frac{N}{4}}+\lambda\left(\frac{1}{2^{\#}}-\frac{1}{2^{*}}\right) \int_{\Omega}|u|^{2^{*}}-\left(1-\frac{1}{2^{\#}}\right)\|g\|_{\eta}\left(\int_{\Omega}|u|^{2^{*}}\right)^{\frac{1}{2^{*}}} .
\end{aligned}
$$

Let $z(x):=\lambda\left(\frac{1}{2^{\#}}-\frac{1}{2^{*}}\right) x-\left(1-\frac{1}{2^{\#}}\right)\|g\|_{\eta} x^{1 / 2^{*}}$. Since the minimum value of $z(x)$ for positive $x$ is $-K$, we get a contradiction. Thus, $v_{j}=0$ for every $j \in J$. Consequently, $u_{n} \rightarrow u$ in $L^{2^{*}}(\Omega)$. Exploiting the complete continuity of the inverse biharmonic operator, we can now show that $u_{n} \rightarrow u$ in $E$.

Working as in Lemma 3.1 in [11], we have

Lemma 7. There exist constants $r, \delta>0$ such that if $\|g\|_{\eta}<\delta$, then $\Psi_{\lambda}(u)>0$ for all $\|u\|_{E}=r$.

Proof. By the Hölder and the Sobolev inequalities, we have that

$$
\begin{aligned}
\Psi_{\lambda}(u) & \geq \frac{1}{2} \int_{\Omega}(\Delta u)^{2} d x-\frac{\lambda}{2^{*}} \int_{\Omega}|u|^{2^{*}} d x-\|g\|_{\eta}\|u\|_{2^{*}} \\
& \geq \frac{1}{2} \int_{\Omega}(\Delta u)^{2} d x-\frac{\lambda}{2^{*} S^{2^{*} / 2}}\left(\int_{\Omega}(\Delta u)^{2} d x\right)^{\frac{2^{*}}{2}}-\|g\|_{\eta} S^{\frac{1}{2}}\left(\int_{\Omega}(\Delta u)^{2} d x\right)^{\frac{1}{2}} .
\end{aligned}
$$

Define $k(x):=\frac{1}{2} x^{2}-\frac{\lambda}{2^{*}} S^{-2^{*} / 2} x^{2^{*}}-\|g\|_{\eta} S^{1 / 2} x, x>0$. It is easy to see that there exists $\delta>0$ such that if $0<\|g\|_{\eta}<\delta$, then $k($.) has a positive maximum attained at a point $r=r\left(\|g\|_{\eta}\right)>0$. Consequently, $\Psi_{\lambda}(u)>0$ for every $u \in E$, with $\|u\|_{E}=r$.

Proof of Theorem 3. Without loss of generality, we may assume that $0 \in \Omega$ and $g(0)>0$. By taking $\varepsilon>0$ small enough, we have that

$$
\int_{\Omega} g u_{\varepsilon}>0,
$$

where $u_{\varepsilon}$ is defined by (20) with $x_{0}=0$. Equation (19) implies that $\Psi_{\lambda}\left(t u_{\varepsilon}\right)<0$ for small $t>0$. Thus,

$$
\inf \left\{\Psi_{\lambda}(u):\|u\|_{E} \leq r\right\}<0
$$

We now choose $g$ so that $0<\|g\|_{\eta}<\delta$ and $\frac{2 \lambda}{N}\left(\frac{S}{\lambda}\right)^{N / 4}-K \geq 0$ (see (21)). An application of the Ekeland variational principle provides a solution to (18).

5. Bifurcation from the principal eigenvalue. Let $\varepsilon>0$ and $\gamma \in(0,1)$. We say that $\Omega$ is $\varepsilon$-close in $C^{4, \gamma}$-sense to the unit ball $B(0,1)$ if there exists a surjective mapping $g \in C^{4, \gamma}(\bar{B}(0,1), \bar{\Omega})$ such that

$$
\|g-I d\|_{C^{4, \gamma}(\bar{B}(0,1), \bar{\Omega})} \leq \varepsilon
$$


THEOREM 8. There is $\varepsilon_{2, N}>0$ such that if $\Omega$ is $\varepsilon$-close in the $C^{4, \gamma}$-sense to $B(0,1)$, with $\varepsilon<\varepsilon_{2, N}$, then the eigenfunction $\varphi_{1, \Omega}($.$) for the first eigenvalue \lambda_{1}$ of

$$
\left.\begin{array}{c}
\Delta^{2} \varphi=\lambda \varphi \text { in } \Omega, \\
u=0, \nabla u=0 \text { on } \partial \Omega
\end{array}\right\}
$$

is unique up to normalization and there exists $c>0$ such that $\varphi_{1, \Omega}(x) \geq c d(x, \partial \Omega)^{2}$.

For more details, we refer to [10].

We assume that our perturbation term $h$ satisfies the following:

(h) $h: \bar{\Omega} \times\left[\lambda_{1}-d, \lambda_{1}+d\right] \rightarrow R$ is continuous with $h_{\infty}=\sup \{|h(x, \lambda)|:(x, \lambda) \in \bar{\Omega} \times$ $\left.\left[\lambda_{1}-d, \lambda_{1}+d\right]\right\}$ and

$$
\int_{\Omega} h\left(x, \lambda_{1}\right) \varphi_{1, \Omega}^{2^{*}}(x) d x \neq 0 .
$$

Definition 9. Let $\digamma: X \rightarrow X^{*}$ be an operator on the real reflexive Banach space $X$. The operator $\digamma$ is said to satisfy the local $\left(S^{+}\right)$property on the set $G \subseteq X$ if any sequence $\left\{x_{n}\right\}_{n \in N}$ in $G$ with $x_{n} \rightarrow x$ weakly in $X$ and $\limsup _{n \rightarrow+\infty}\left\langle\digamma\left(x_{n}\right), x_{n}-x\right\rangle \leq 0$ satisfies $x_{n} \rightarrow x$ strongly in $X$.

We define the operators $J, S, H_{\lambda}: E \rightarrow R$ with the use of the duality pairing in $E$ :

$$
\begin{gathered}
(J(u), v)=\int_{\Omega} \Delta u \Delta v, \\
(S(u), v)=\int_{\Omega} u v
\end{gathered}
$$

and

$$
\left(H_{\lambda}(u), v\right)=\int_{\Omega}|\nabla u|^{p-2} \nabla u \nabla v+\int_{\Omega} h(x, \lambda)|u|^{2^{*}-2} u v .
$$

It is clear that $u \in E$ is a (weak) solution to (2) if and only if $u$ solves the operator equation:

$$
N_{\lambda}(u):=J(u)+\lambda S(u)-H_{\lambda}(u)=0 .
$$

Lemma 10. Suppose that $\rho_{0}<\min \left\{1, h_{\infty}^{-(N-4) / 8} S^{N / 8}\right\}$. Then, $N_{\lambda}($.$) satisfies the local$ $\left(S^{+}\right)$property in $B\left(0, \rho_{0}\right)$.

Proof. Let $\left\{u_{n}\right\}_{n \in N}$ be a sequence in $B\left(0, \rho_{0}\right)$. By passing to a subsequence, if necessary, we may assume that $u_{n} \rightarrow u_{0}$ weakly in $E$. Furthermore, let

$$
\limsup _{n \rightarrow+\infty} N_{\lambda}\left(u_{n}\right)\left(u_{n}-u_{0}\right) \leq 0
$$

that is,

$$
\begin{gathered}
\limsup _{n \rightarrow+\infty}\left\{\int_{\Omega} \Delta u_{n} \Delta\left(u_{n}-u_{0}\right)+\int_{\Omega}\left|\nabla u_{n}\right|^{p-2} \nabla u_{n} \nabla\left(u_{n}-u_{0}\right)\right. \\
\left.-\lambda \int_{\Omega} u_{n}\left(u_{n}-u_{0}\right)-\int_{\Omega} h(x, \lambda)\left|u_{n}\right|^{2^{*}-2} u_{n}\left(u_{n}-u_{0}\right)\right\} \leq 0 .
\end{gathered}
$$


Note that, by (24),

$$
\begin{gathered}
\int_{\Omega} \Delta u_{n} \Delta u_{0} \rightarrow \int_{\Omega}\left(\Delta u_{0}\right)^{2}, \\
\int_{\Omega}\left|\nabla u_{n}\right|^{p-2} \nabla u_{n} \nabla\left(u_{n}-u_{0}\right) \rightarrow 0, \\
\int_{\Omega} u_{n}\left(u_{n}-u_{0}\right) \rightarrow 0
\end{gathered}
$$

and

$$
\int_{\Omega} h(x, \lambda)\left|u_{n}\right|^{2^{*}} \rightarrow \int_{\Omega} h(x, \lambda)\left|u_{0}\right|^{2^{*}}+\int_{\Omega} h(x, \lambda) d \widetilde{v},
$$

where $\widetilde{v}=\sum_{j \in J} v_{j} \delta_{x_{j}}$. Since the sequence $\left\{\left|u_{n}\right|^{2^{*}-2} u_{n}\right\}_{n \in N}$ is bounded in $\left(L^{2^{*}}(\Omega)\right)^{\prime}$, we have that, up to a subsequence, $\left|u_{n}\right|^{2^{*}-2} u_{n} \rightarrow\left|u_{0}\right|^{2^{*}-2} u_{0}$ weakly in $\left(L^{2^{*}}(\Omega)\right)^{\prime}$. Thus,

$$
\int_{\Omega} h(x, \lambda)\left|u_{n}\right|^{2^{*}-2} u_{n} u_{0} \rightarrow \int_{\Omega} h(x, \lambda)\left|u_{0}\right|^{2^{*}}
$$

In view of hypothesis $(h),(24)$ and (33)-(37), (32) yields

$$
\tilde{\mu}(\bar{\Omega}) \leq h_{\infty} \widetilde{v}(\bar{\Omega})
$$

where $\tilde{\mu}=\sum_{j \in J} \mu_{j} \delta_{x_{j}}$, and by exploiting (24) again, we get

$$
\widetilde{\mu}(\bar{\Omega}) \leq h_{\infty} S^{-\frac{2^{*}}{2}} \widetilde{\mu}(\bar{\Omega})^{\frac{2^{*}}{2}} .
$$

Consequently, $\tilde{\mu}(\bar{\Omega})=0$ or $h_{\infty}^{-(N-4) / 4} S^{N / 4} \leq \tilde{\mu}(\bar{\Omega})$. If $h_{\infty}^{-(N-4) / 4} S^{N / 4} \leq \tilde{\mu}(\bar{\Omega})$, then, since $\left\|u_{n}\right\|_{E}<\rho_{0}$, we should have $\tilde{\mu}(\bar{\Omega})<\rho_{0}^{2}<h_{\infty}^{-(N-4) / 4} S^{N / 4}$, a contradiction. Consequently, $\tilde{\mu}=0$. In view of (24) and the strict convexity of $E$, we get that $u_{n} \rightarrow u$ in $E$.

In view of Lemma 10 and Theorem 1.6 in [7], the degree $\operatorname{Deg}\left(N_{\lambda}, D, 0\right)$ is well defined for all open, bounded and nonempty sets $D \subset B\left(0, \rho_{0}\right)$ whenever $0 \notin N_{\lambda}(\partial D)$. Define

$$
\widetilde{N}_{\lambda}(u):=J(u)+\lambda S(u) .
$$

The degree $\operatorname{Deg}\left(\widetilde{N}_{\lambda}, B(0, \rho), 0\right)$, for any $0<\rho<\rho_{0}$, is also well defined for $\lambda \in\left(\lambda_{1}-\right.$ $\left.d, \lambda_{1}+d\right), \lambda \neq \lambda_{1}$,

$$
\operatorname{Deg}\left(\tilde{N}_{\lambda}, B(0, \rho), 0\right)=1, \lambda \in\left(\lambda_{1}-d, 0\right)
$$

and

$$
\operatorname{Deg}\left(\widetilde{N}_{\lambda}, B(0, \rho), 0\right)=-1, \lambda \in\left(0, \lambda_{1}+d\right) .
$$

For more details, we refer to [3, 7].

The proof of the following lemma follows as an easy combination of Hölder's inequality with the Sobolev embeddings and it is omitted. 
LEMMA 11. The operator $H_{\lambda}($.$) satisfies$

$$
\lim _{\|u\|_{E} \rightarrow 0} \frac{\left\|H_{\lambda}(u)\right\|_{E^{*}}}{\|u\|_{E}}=0
$$

uniformly for $\lambda$ in a bounded subset of $R$.

By exploiting the previous lemma and the homotopy invariance property of the degree, we get that for every $\lambda \in\left(\lambda_{1}-d, \lambda_{1}+d\right), \lambda \neq \lambda_{1}$, there exists $\rho>0$ such that

$$
\operatorname{Deg}\left(N_{\lambda}, B(0, \rho), 0\right)=1, \lambda \in\left(\lambda_{1}-d, 0\right)
$$

and

$$
\operatorname{Deg}\left(N_{\lambda}, B(0, \rho), 0\right)=-1, \lambda \in\left(0, \lambda_{1}+d\right)
$$

Note that the index of the isolated zero of $N_{\lambda}$ changes by magnitude 2 when $\lambda$ crosses $\lambda_{1}$, so working as in Theorem 1.3 and Corollary 1.12 in [14] we get

THEOREM 12. Equation (2) admits a continuum $C$ of nontrivial solutions $(\lambda, u) \subseteq$ $R \times E$ bifurcating from $\left(\lambda_{1}, 0\right)$, which meets the boundary of $\left[\lambda_{1}-d, \lambda_{1}+d\right] \times B\left(0, \rho_{0}\right)$.

REMARK 13. Most of the above results can be extended to the case of the equation $\Delta^{2} u+\Delta_{p} u=\lambda|u|^{s-2} u$ with Dirichlet boundary conditions.

\section{REFERENCES}

1. H. Brezis, Analyse fonctionnelle-Theorie et applications (Masson, Paris, 1983, 1993), (Dunod, Paris, 1999).

2. H. Brezis and L. Nirenberg, Positive solutions of nonlinear elliptic equations involving critical Sobolev exponents, Commun. Pure Appl. Math. XXXVI (1983), 437-477.

3. J. Chabrowski, P. Drabek and E. Tonkes, Asymptotic bifurcation results for quasilinear elliptic operators, Glasgow Math. J. 47 (2005), 55-67.

4. J. Chabrowski and J. M. do Ó, On some fourth-order semilinear elliptic problems in $R^{N}$, Nonlin. Anal. TMA 49 (2002), 861-884.

5. J. Chabrowski and J. Yang, Nonnegative solutions for semilinear biharmonic equations in $R^{N}$, Analysis 17 (1997), 35-59.

6. Q-H. Choi and T. Jung, Positive solutions on nonlinear biharmonic equation, Kangweon-Kyungki Math. J. 5(1) (1997), 29-33.

7. P. Drabek, A. Kufner and F. Nicolosi, Quasilinear elliptic equations with degenerations and singularities (W. de Gruyter, Berlin, 1997).

8. D. E. Edmunds, D. Fortunato and E. Jannelli, Critical exponents, critical dimensions and the biharmonic operator, Arch. Rational Mech. Anal. 112 (1990), 269-289.

9. N. Ghoussoub, Duality and perturbation methods in critical point theory (Cambridge University Press, Cambridge, 1993).

10. H.-C. Grunau and G. Sweers, The maximum principle and positive principal eigenfunctions for polyharmonic equations, in Reaction-Diffusion Systems (Trieste, 1995), Lect. Notes Pure Appl. Math. 194 (Dekker, New York, 1998), 163-182).

11. M. Guedda, On nonhomogeneous biharmonic equations involving critical Sobolev exponent, Portugaliae Math. 56 Fasc. 3 (1999), 299-308.

12. T. Jung and Q-H. Choi, An application of a variational linking theorem to a non-linear biharmonic equation, Nonlinear Anal. TMA 47 (2001), 3695-3705.

13. P. L. Lions, The concentration-compactness principle in the Calculus of Variations. The limit case, I, II. Rev. Mat. Iberoamer. 1(1) (1985), 145-201 and 1(2) (1985), 45-121. 
14. P. H. Rabinowitz, Some global results for nonlinear eigenvalue problems, J. Funct. Anal. 7 (1971), 487-513.

15. R. C. A. M. Van Der Vorst, Fourth order elliptic equations with critical growth, $C . R$. Acad. Sci. Paris Sér. I Math. 320(3) (1995), 295-299.

16. G. Xu and J. Zhang, Existence results for some fourth-order nonlinear elliptic problems of local superlinearity and sublinearity, J. Math. Anal. Appl. 281 (2003), 633-640. 